\title{
Automatic multi-trajectory planning solution for steerable catheters
}

\author{
Alberto Favaro*a ${ }^{*}$ Leonardo Cerri ${ }^{a}$, Davide Scorza $^{a, b}$ and Elena De Momi ${ }^{a}$
}

\begin{abstract}
The present work describes a novel approach to trajectory planning for minimally invasive surgery consisting of an algorithm able to provide the surgeon with multiple curvilinear paths to connect an entry area defined on the brain cortex to a specific target point in the brain. A criterion based on the minimum distance from the safety-critical brain structures (blood vessels, thalamus and ventricles) is used to rank the obtained trajectories. The solution is integrated onto the EDEN2020* $^{*}$ programmable bevel-tip needle, a multi-segment probe whose steering ability derives from the offset generated on its tip, and provides a level of tolerance with respect to tracking errors arising from catheter model inaccuracies. The case of study of the work consists of a typical Deep Brain Stimulation scenario where tests have been performed in order to compare the result obtained from standard rectilinear trajectory planning against this novel curvilinear solution using the clearance from obstacles as an index of performance of the estimated solutions.
\end{abstract}

\section{INTRODUCTION}

Parkinson's disease (PD) represents one of the most common neurodegenerative disorders with a prevalence of $\sim 0.3 \%$ in industrialized countries and a peak of $1 \%$ in people over the age of 60 [1].

A surgical approach capable of improving patients dailyliving activities by reducing PD symptoms is represented by Deep Brain Stimulation (DBS), an adjustable and reversible alternative to direct brain tissue ablation based on electrical stimuli delivered by implantable electrodes [2], [3]. Generally, the anatomical target of DBS consists of the subthalamic nuclei (STN) [2].

Estimating a safe insertion pathway to reach STN can be complex and time consuming for surgeons, who may spend up to $1 \mathrm{~h} 30 \mathrm{mins}$ performing this task [3]. Moreover, even careful planning can lead to surgery-related complications such as hemorrhaging, seizures or incorrect electrode placement [4]. Solutions able to automate the definition of DBS trajectories can prove useful in assisting the surgeon in this task, not only by reducing the time required by this step, but also by tuning the solutions in accordance with optimality criteria, such as the reduction of the total path length and the clearance from safety-critical brain structures (i.e blood vessels, lateral ventricles, thalamus and cerebellum [5]). Obstacle-avoidance capability plays a key role in preventing

\footnotetext{
*This project has received founding from the European Unions Horizon 2020 research and innovation program under grant agreement No 688279.

${ }^{a}$ Department of Electronics, Information and Bioengineering, Politecnico di Milano, Milan, Italy alberto.favaro@polimi.it elena.demomi@polimi.it

${ }^{b}$ e-Health and Biomedical Applications Department, Vicomtech-IK4, Donostia-San Sebastian, Spain and Biodonostia Health Research Institute, Donostia-San Sebastian, Spain dscorza@vicomtech.org 978-1-5386-2512-5/18\$31.00 @2018 IEEE
}

DBS-related complications, so the possibility to exploit electrodes able to steer along curvilinear trajectories to reach a specific brain area as STN can overcome limitations linked to straight paths and rigid embodiments. Different prototypes of steerable needles for Minimally Invasive Surgery (MIS) were proposed in literature and some of them find possible implementation in DBS [6], [7]. Specifically, the present work will be focused on the EDEN2020* (www.eden2020.eu) programmable bevel-tip needle $(\mathrm{PBN})$. This probe is made of four interlocked sections (a thorough representation of PBN design can be found in [8], [9]) and its steering ability originates from the offset determined at the needle tip by the reciprocal displacement among the sections. As it was originally intended as a means for drug delivery in glioblastomas treatment, PBN includes inner working channels that are suitable for being used for electrode deployment, making PBN potentially appropriate for DBS implantation. A previous work [10] describes an automatic 3D planner for curvilinear trajectories in MIS designed to guarantee the clearance from safety-critical brain structures accounting for kinematic constraints and non-holonomicity of PBN as well as for possible inaccuracies in catheter modeling. The algorithm, developed as a module for 3D Slicer@ (www.slicer.org), uses the asymptotically-optimum solution described in [11] to connect an entry point (EP) to a target point (TP), solving a so-called single-query planning task and performs a path optimization in order to meet PBN constraints.

In the present work we propose a planning tool based on the afore-mentioned planning algorithm, able to provide the surgeon with a pool of feasible trajectories resulting from a multi-query planning process which uses a DBS scenario as case of study.

The paper is structured as follows. In Section II, an overview of the current approaches to MIS path planning is provided. Section III describes the proposed solution and the dataset used. Results from simulations are presented in Section IV while discussion and conclusions can be found in Section V.

\section{RELATED WORKS}

Several automatic approaches were proposed for straight trajectory planning in MIS applications. Briault et al. in [12] suggested a DBS planning framework for STN targeting which exploits a multi-modal Magnetic Resonance Imaging (MRI) acquisition protocol to estimate the best entry area over the whole brain cortex for straight electrodes insertion, guaranteeing proper avoidance of safety-critical inner structures. Essert et al. [3] formalized the implicit and explicit principles used by surgeons in DBS manual planning to tune 
an automatic straight trajectory planner.

A multi-planner for Stereoelectroencephalography (SEEG) solution proposed in [5] (subsequently improved in [13]) is able to provide the surgeon with the best configuration for multiple linear electrodes, accounting for the spacial relationships among them. This is also obtained by expanding each EP initially selected by the surgeon to an entry area, where new EPs are estimated in order to search for novel trajectories. The latter approach is also used in a multi-trajectory planner for keyhole neurosurgery [14], where the best straight trajectory is defined according to specific optimality criteria.

Regarding curvilinear trajectory, a variety of solutions can be found in MIS. Duindam et al. [15] proposed a method based on inverse catheter kinematics which has showed limited obstacle-avoidance capability when tested in a simplified simulation environment. In [16], Park et al. describe an algorithm for MIS applications based on a probability map, but without testing it in the presence of obstacles.

The discretization of the working domain is the key feature of the Adaptive Fractal Tree [17], which uses fractal theory and process parallelization to achieve a computational time compatible with real-time replanning, but requires a performing Graphics Processing Unit.

Sampling-based solutions are the current trend for generic single-query, path planning problems. In [18], a combination of Rapidly-exploring Random Tree (RRT) and reachabilityguided approach (RG-RRT) is described. The method can compute curvilinear trajectories in complex 3D scenarios by sequentially connecting arcs with bounded curvature. The short time required for computation allows its use in realtime applications, even though tests were only performed in simplified 3D environments. A similar approach was presented by Caborni et al. [19] for 2D trajectory planning. In the Batch Informed Tree (BIT*) suggested by Gammel et al. in [11], the best trajectory in terms of path length is found by increasingly sampling the working domain, confining the research within an ellipsoidal region. As the research for the global optimum proceeds, the ellipsoid decreases its volume proportionally with the shortening of the path length, focusing the research to a smaller region. Authors demonstrated the asymptotic optimality of the method and experiments showed a reduction in the computational time, even though no assessment was performed in MIS scenarios. In a previous work [10], an approach similar to BIT* was implemented for developing an automatic 3D planner, which showed good planning capability in a typical neurosurgical scenario, but also highlighted the effect of catheter curvature limitation in hindering the definition of viable trajectories. This work aims to increase the probability of finding at least one solution for the planning task by expanding the original single-query planning problem (a single EP to be connected to a single TP) to a multi-query task (multiple EPs-one TP) through the definition of an entry area and a set of EPs in the neighborhood of the original EP defined by the surgeon, which are sequentially provided to the planning algorithm. The method was compared with the standard rectilinear approach through an analysis of the differences in terms of clearance from obstacles between the two methods. The performances were additionally put in relation with the control accuracy over the insertion process leading to possible trajectory tracking error and which, in the case of the steerable needle, derives from inaccuracies in the catheter modeling.

\section{METHODS}

Two MRI acquisition protocols are used: a T1-weighted volumetric acquisition (Philip Ingenia CX 3T, TR/TE (ms): 12/5.8, data matrix: $320 \times 299$, FOV $(\mathrm{mm}): 256 \times 240$, inplane resolution $(\mathrm{mm}): 0.80 \times 0.80$, thickness $(\mathrm{mm}): 0.80$, number of sections: 236) for morphological characterization and a 3D high-resolution time-of-flight (TOF) acquisition (Philip Ingenia CX 3T, TR/TE (ms): 23/3.5, acquisition plane: axial, data matrix: $500 \times 399$, FOV $(\mathrm{mm}): 200 \times 200$, in-plane resolution $(\mathrm{mm}): 0.40 \times 0.50$, thickness $(\mathrm{mm}): 0.90$, number of sections: 210) for arterial vessels visualization. Acquisitions from one healthy subject were performed at the Center of Excellence for High Field Magnetic Resonance (CERMAC), Ospedale San Raffaele, Milan, Italy under ethical approval n. 80/INT/2016 and patient gave written informed consent. A Computed Tomography (CT) acquisition (resolution $(\mathrm{mm})$ : $0.43 \times 0.43 \times 2.52$ ) is used to reconstruct the skull surface. A non-rigid registration is performed on the MRI and CT dataset using the Elastix toolbox [20].

A schematic representation of the workflow for the proposed solution is depicted in Fig. 1 and described hereinafter.

\section{A. MRI data elaboration}

MRI data are elaborated in order to obtain the required morphological information: 3D Slicer is used to segment brain ventricles from the $\mathrm{T} 1$ series via thresholding and the same method is used to obtain the arterial tree from the TOF imaging and the patient's skull from CT data.

The STN and the thalamus are identified in patient anatomy by making use of an on-line brain atlas [21], after a nonrigid co-registration performed via Elastix toolbox. Freesurfer pipeline [22] is executed on T1 series in order to obtain the cortical surface and other related indexes. Specifically, the curvature index is used to separate the original mesh $\mathcal{M}$ in gyri and sulcis $\left(\mathcal{M}_{g}, \mathcal{M}_{s}\right)$.

\section{B. Entry area definition and mesh decimation}

As the cortical surface has been divided in $\mathcal{M}_{g}$ and $\mathcal{M}_{s}$, the surgeon is asked to roughly identify a preferred entry location for the catheter and to place a fiducial point $\left(\mathrm{EP}_{\text {init }}\right)$ over $\mathcal{M}_{g}$. At this phase, $\mathcal{M}_{s}$ is removed from the pool of possible entry locations: in this way, the probability to hit a vessel at the EP location is reduced as vessels are more likely located at the bottom of sulcis [23]. Similarly, the target location (TP) is manually placed over the STN, in the same hemisphere of $\mathrm{EP}_{\text {init }}$.

As the query (i.e. the couple $\mathrm{EP}_{\text {init }}$-TP) is defined, a circular 


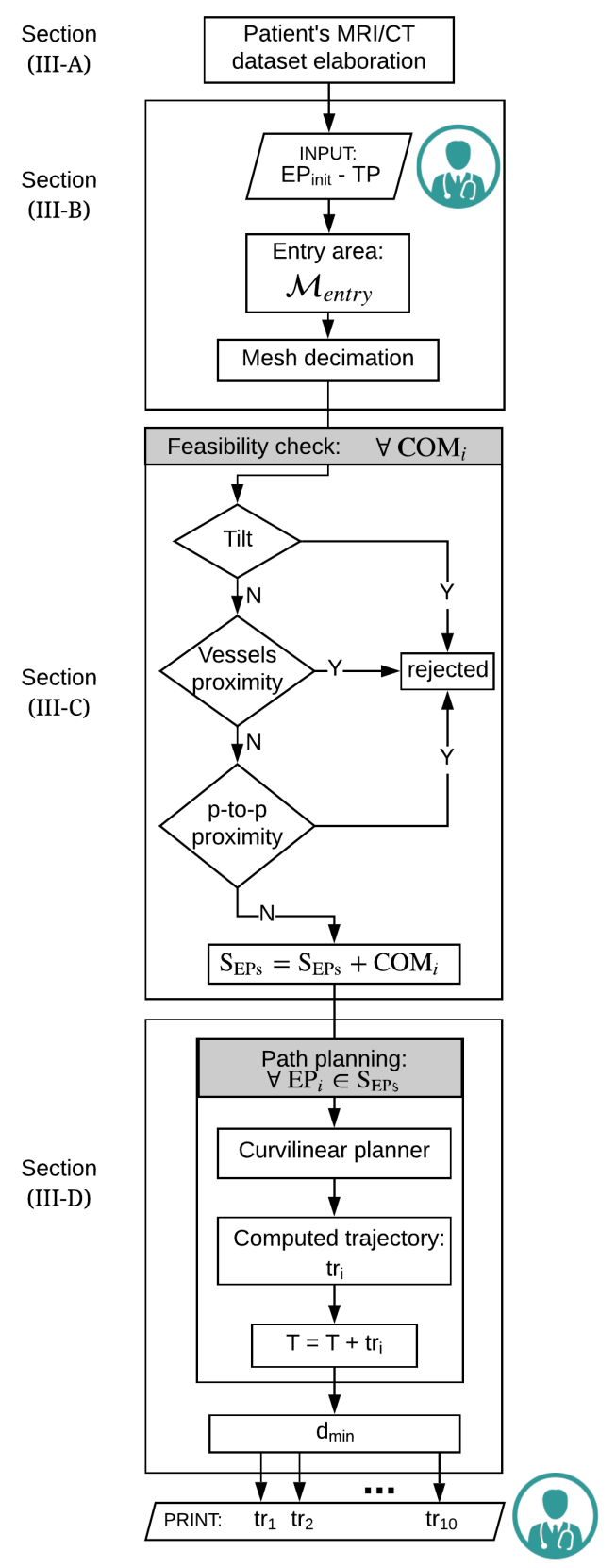

Fig. 1: Schematic representation of the workflow for the presented solution.

area $\mathcal{M}_{\text {entry }} \subset \mathcal{M}_{g}$ is built such that:

$$
\left\|\mathrm{EP}_{\text {init }}-\mathbf{v}\right\|<r \quad \forall \mathbf{v} \in \mathcal{M}_{\text {entry }}
$$

where $\mathbf{v}$ is the 3D position of a generic vertex of $\mathcal{M}_{\text {entry }}$ and $r$ consists in the desired radius of the circular area. $\mathcal{M}_{\text {entry }}$ represents the region where to perform the search for new possible EPs.

Subsequently, a mesh decimation is performed using the approach proposed by Schroeder et al. [24] in order to reduce the number of vertices composing the mesh and, consequently, the pool of possible new EPs. The undersampling is implemented through the VTK scientific library (Kitware, Clifton Park, NY, USA).

\section{Points feasibility check}

For each triangle $i$ composing the decimated mesh, the center of mass is computed $\left(\mathrm{COM}_{i}\right)$ and a multiple check is performed to ensuring that this point corresponds to a feasible new catheter EP:

1) Tilt check: the entry angle over the skull aims to resemble the normal direction of the skull as closely as possible, otherwise the drill may slip over the skull making difficult to create the borehole [25]. To ensure this requirement is met, the normal direction $\vec{n}_{i}$ is computed for $\mathrm{COM}_{i}$ over the cortical surface. By projecting $\mathrm{COM}_{i}$ on the external skull surface along $\vec{n}_{i}$, it is possible to define the corresponding drilling point over the skull $\left(\mathrm{COM}_{i}^{S}\right)$.

$\mathrm{COM}_{i}$ is rejected from the pool of possible new EPs if:

$$
\angle\left(\vec{n}_{i}, \vec{n}_{i}^{S}\right)>\alpha_{\max }
$$

where $\vec{n}_{i}^{S}$ represent the normal direction computed over $\operatorname{COM}_{i}^{S}$ and $\alpha_{\max }$ is the maximum inclination admitted for the drill with respect to the skull surface. Both $\vec{n}_{i}$ and $\vec{n}_{i}^{S}$ result from averaging the normal directions over their adjacent vertices of the relative meshes.

2) Vessel proximity check: only if the previous test has been passed, $\mathrm{COM}_{i}$ is evaluated for its vicinity to superficial blood vessels which can lie underneath $\mathrm{COM}_{i}$. In order to achieve this, the minimum steering space $l_{\min }$ required by the catheter to avoid an obstacle placed along its insertion direction is computed, as showed in Fig. 2a, by considering the PBN curvature limit $\left(K_{P B N}\right)$ and the maximum diameter $\oslash_{\text {angio }}$ of pial blood vessels [26]. A straight cylindrical volume $V^{\text {safe }}$ is generated, it spans from $\mathrm{COM}_{i}$ toward $\mathrm{TP}$ with a height $V_{h}^{\text {safe }}=l_{\min }$ and a diameter $V_{\oslash}^{\text {safe }}=\oslash_{P B N}$. The presence of vessels within $V_{\text {safe }}$ is checked, which determines the rejection of $\mathrm{COM}_{i}$ from the poll of possible new EPs. A representation of $V^{s a f e}$ is proposed in Fig. 2b.

3) Point-to-point proximity check: in case $\mathrm{COM}_{i}$ lies too close to another $\mathrm{COM}$ (i.e. $\left\|\mathrm{COM}_{i}-\mathrm{COM}_{j}\right\|<d_{p p}$ ), $\mathrm{COM}_{i}$ is rejected.

Tilt, vessel and point-to-point proximity represent the three strong constraints a COM must meet to be acknowledged as candidate EPs. COMs that pass all these checks are added to a set $S_{E P s}$ composed by new feasible EPs for catheter implantation.

\section{Path planning}

$S_{E P s}$, the set of feasible EPs, goes through the automatic planning phase: each $\mathrm{EP} \in S_{E P S}$ is provided sequentially to the single-query curvilinear path planner described in [10]. The planner, suited on PBN kinematics and briefly described hereinafter, exploits an heuristic sample-based approach [11] which allows to speed up the research for a solution to the query-problem by focusing the research to a subspace of the working domain. 


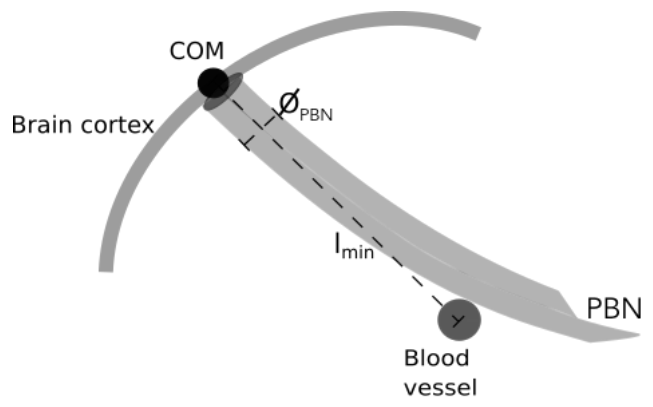

(a)

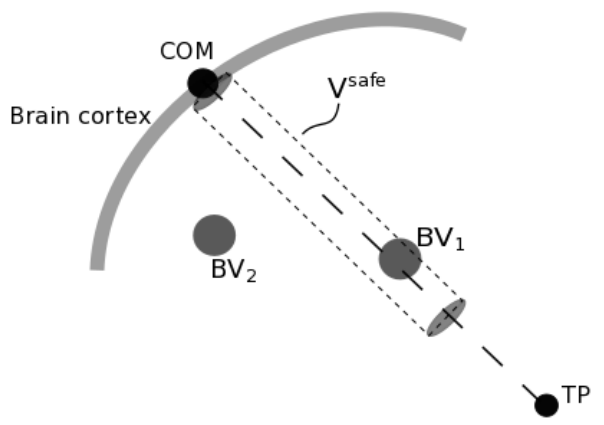

(b)

Fig. 2: Illustration of the vessel proximity check. In Fig. 2a, a representation of $l_{\min }$ as the minimum distance required by the catheter to steer is showed, which is determined considering the maximum diameter of pial blood vessels and the maximum curvature admitted by PBN. Fig. 2b showed the construction of $V_{s a f e}$ as a cylindrical volume having $l_{\text {min }}$ as principal axis and $\oslash_{P B N}$ as diameter. In this case, the vessel $B V_{2}$ is outside $V_{\text {safe }}$ whilst $B V_{2}$ lies inside and thus determines the rejection of the COM from the pool of new EPs.

1) First path definition: an initial ellipsoidal volume is built around EP and TP and its volume is sampled with points which are gradually added to a oriented graph guaranteeing obstacle avoidance. As the number of samples increase, the ellipsoid enlarges proportionally until a first path from EP to TP is found.

2) Path optimization: New points are sampled in the ellipsoidal volume and the graph pruned to reduce the length of the path. Each time a shorter path is found, the ellipsoid reduces, focusing the research to a smaller subspace of the working domain. When the density of samples reach a threshold without further reduction of the path length, the latest path is considered as the best solution to the planning problem.

3) Interpolation and smoothing: The best path undergoes an Hermite spline interpolation to provide $\mathcal{C}^{2}$ continuity and a smoothing step to reduce its curvature and meet PBN curvature limit.

4) Uncertainty margin: to account for catheter modeling inaccuracies which can result in error in trajectory tracking over the insertion procedure, a safety margin is add to the estimated trajectory. This consists of a conical-shaped volume that enlarges in the 3D space approaching to the TP according with the standard deviation of the control error $\mathbf{m} \sim \mathcal{N}(0, \mathbf{M})$. The uncertainty margin represents a further hard constraint for the curvilinear path planner and it is taken into consideration when determining if the estimated trajectory guarantees obstacle clearance. In case the margin intersects a safety-critical structure, the trajectory is rejected and no solution is provided for the specific couple EP-TP.

As the planning algorithm is run over all $\mathrm{EP} \in S_{E P s}$, the estimated trajectories are evaluated according to the safety level they provide in term of clearance from critical brain structures. To this scope, the minimum distance from an obstacle is computed for each trajectory $\operatorname{tr} \in T$, where $T$ represent the set of all the trajectories obtained by the planning process, and the surgeon in then provided with the ten best trajectories according to this metric. A representation of the trajectories obtained by the planning process is presented in Fig. 3.

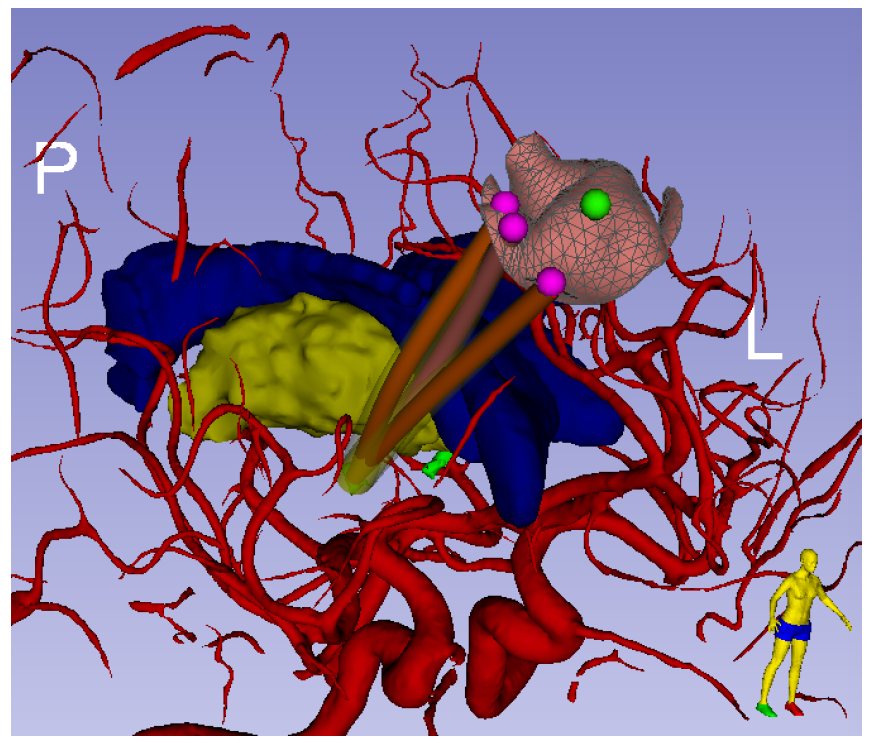

Fig. 3: Results of the curvilinear planning process. The brain angiography, the thalamus, the ventricles and the STN are showed respectively in red, olive, blue and light green. EP $P_{\text {init }}$ and the new EPs are displayed over the pink entry area in light green and purple respectively. The estimated trajectory, in red, starts from the EPs and ends on the STN (not visible). The uncertainty margin is presented in dark yellow as a cone that surrounds the estimated trajectories.

\section{RESULTS}

To check the feasibility of the proposed method, simulations were performed on an iMac (OS-X 10.11.6, 3.1GHz Intel Core i7, 8GB RAM). To this scope, the dataset described in Section III-A was used. The 2 ideal TPs over the right and left STN as well as 6 candidate $\mathrm{EP}_{\text {init }}, 3$ on the right and 3 on the left hemisphere, have been identified. The entry areas $\left(\mathcal{M}_{\text {entry }}^{i}, i=1,2, \ldots, 6\right)$, whose representation is showed in Fig. 4, were computed following the steps described in Sections III-B, III-C.

\section{A. Decimation threshold}

To find the mesh decimation parameter $\left(\xi_{\text {opt }}\right.$, see Section III-B) able to guarantee the greatest number of detected 


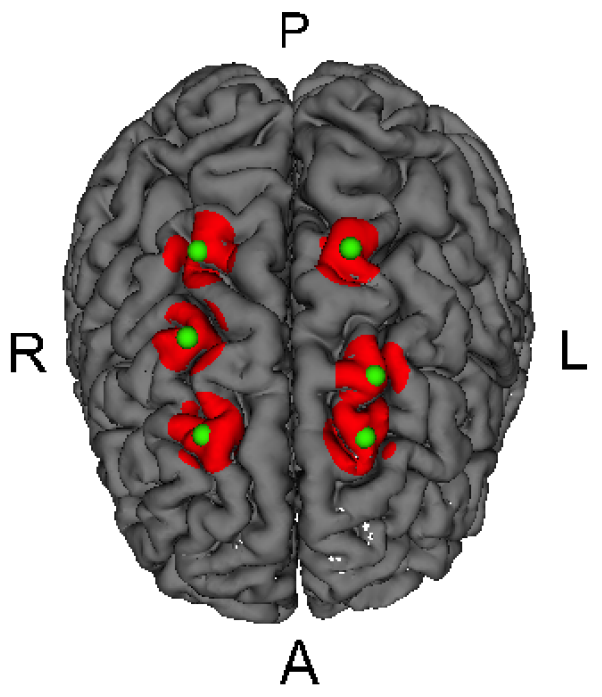

Fig. 4: Entry areas identified over the brain cortex (in red), 3 on the left and 3 on the right hemisphere. The manually-selected entry points are displayed in green at the center of the circular areas.

COMs, a test was carried out to evaluate the number of COMs which meet the geometric and safety constraints described in Section III-C. Each of the six $\mathcal{M}_{\text {entry }}$ underwent a gradual decimation process performed by decremental steps $\Delta_{\xi}$ until a saturation in the number of COMs was reached. Simulation parameters and hard constraints values are reported in Table I. The values of the maximum inclination angle $\alpha_{\max }$ and $\mathcal{M}_{\text {entry }}$ radius were consistent with those used in [13], while the minimum distance $d_{p p}$ between adjacent COMs was defined as a reasonable value in accordance with the resolution of the MRI data.

The test demonstrated a saturation in the number of COMs obtained by mesh decimation and the data showed a good fit with an exponential trend $(\mathrm{R}$-square $=0.986)$, thus an optimal decimation value has been defined as $\xi_{\text {opt }}=70 \%$. This decimation level was used in the subsequent simulations.

TABLE I: Values used in the simulation of Section IV-A for parameters and hard constraints.

\begin{tabular}{llll}
\hline \multicolumn{2}{c}{ Hard constraints } & \multicolumn{2}{c}{ Simulation parameters } \\
\hline$\alpha_{\max }[\mathrm{deg}]$ & 30 & Decimation range $[\%]$ & $100-65$ \\
$K_{P B N}\left[\mathrm{~mm} \mathrm{~m}^{-1}\right]$ & 0.014 & Decimation step $\Delta_{\xi}[\%]$ & 2.5 \\
$\oslash_{P B N}[\mathrm{~mm}]$ & 2.5 & $\mathcal{M}_{\text {entry }}$ radius $r[\mathrm{~mm}]$ & 10 \\
$\oslash_{\text {angio }}[\mathrm{mm}]$ & 1.2 & & \\
$d_{p p}[\mathrm{~mm}]$ & 2 & & \\
\hline
\end{tabular}

\section{B. Planning}

Next simulation protocol assessed the benefits of curvilinear planning with respect to a rectilinear solution in terms of clearance from obstacles over the variation in catheter control accuracy. The latter is represented by the uncertainty margin which, in turn, features the error in trajectory tracking attributable to inaccuracies in catheter modeling.

To the scope of this test, the sets $S_{E P s}^{i}, i=1,2, \ldots, 6$ constituted by the feasible EPs defined over the 6 entry areas were provided to both the curvilinear and a standard rectilinear planner. Different simulations were run, making the variance of the tracking error $\mathbf{M}$ varying in a range from 0 to a maximum of $2 \times 10^{-3} \mathrm{~mm}^{2}$ ([27] was used as reference) . For each rectilinear and curvilinear trajectory, the minimum distance $\left(d_{\min }\right)$ from the closest safety-critical structure (e.g. a blood vessel, the thalamus or a ventricle) was computed.

In Fig. 5, results from the different simulations are presented where a small, constant value $\mathbf{M}_{\text {rect }}=5 \times 10^{-4} \mathrm{~mm}^{2}$ was assigned to rectilinear trajectories as a more accurate control shall be expected for rigid catheter with respect to the steerable counterparts. An analysis of the results suggests $d_{\text {min }}$ decreases in a linear manner as the variance of the tracking error (M) increases (linear curve fit, Rsquare $=0.979$ ).

Curvilinear solutions produced improved results compared to the rectilinear counterparts when $\mathbf{M}=0 \mathrm{~mm}^{2}$ (no error in trajectory tracking), as can be noticed by the fact that the left-most boxplot stands above the level settled by the rectilinear solutions (depicted in blue in Fig. 5 as median, $25^{\text {th }}$ and $75^{\text {th }}$ percentiles) but performance rapidly worsens for greater values of $\mathbf{M}$.

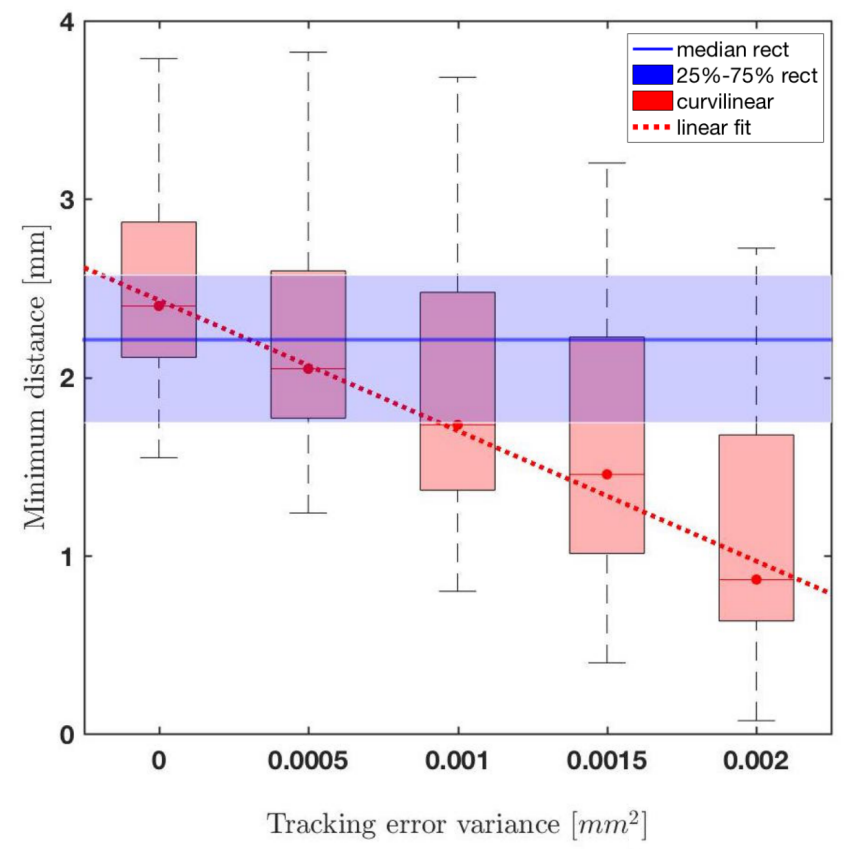

Fig. 5: Graph of the minimum distance from safety-critical obstacles over the variance (M) of the trajectory tracking error.

\section{DISCUSSION AND CONCLUSIONS}

The work presents a comparison between standard straight trajectory planning and a novel curvilinear planner integrated with the EDEN2020 programmable bevel-tip needle, a surgical probe for MIS capable of steering in 3D space by exploiting the offset generated on its tip. In the context of DBS, which represents the field of application of the 
present work, different methods can be found for electrode placement optimization as showed in [3], [12], but these approaches consist of rectilinear solutions and, according to the authors' knowledge, no methods are proposed in literature for curvilinear planning in DBS scenario.

The comparison was carried out using the distance from safety-critical structures as a performance index for paths estimated by the two different approaches. A substantial equivalence is found when an identical level of inaccuracy is assigned to the two planners ( $\mathbf{M}=\mathbf{M}_{\text {rect }}=5 \times 10^{-4} \mathrm{~mm}^{2}$ ) whilst, for greater values of $\mathbf{M}$, the curvilinear solution shows a fast worsening of the performance. This is due to the fact that the uncertainty margin increases its size proportionally with the distance from the catheter EP by a factor $\sqrt{\mathbf{M}}$, the standard deviation of the tracking error, so given the same trajectory length, a greater $\mathbf{M}$ determines a bigger uncertainty margin, reducing the distance from safety-critical structures. In light of these results, two main conclusions can be drawn. Firstly, a small control error (and thus a high accurate catheter modeling which determines good trajectory tracking) can lead curvilinear planning to overtake the performance of standard rectilinear planning solutions in terms of distance from obstacles. In addition, further improvement in this sense can be achieved by developing a curvilinear planner based on different approaches, as the potential field method [28], which is known to maximize obstacle clearance, or a planner that inherently optimizes the trajectory not only for the total path length (as implemented in this work) but also for the distance from brain structures, through the definition of a proper cost function aimed at finding a compromise between these two indexes.

\section{REFERENCES}

[1] L. M. De Lau and M. M. Breteler, "Epidemiology of parkinson's disease," The Lancet Neurology, vol. 5, no. 6, pp. 525-535, 2006.

[2] A. L. Benabid, "Deep brain stimulation for parkinsons disease," Current opinion in neurobiology, vol. 13, no. 6, pp. 696-706, 2003.

[3] C. Essert, C. Haegelen, F. Lalys, A. Abadie, and P. Jannin, "Automatic computation of electrode trajectories for deep brain stimulation: a hybrid symbolic and numerical approach," International journal of computer assisted radiology and surgery, vol. 7, no. 4, pp. 517-532, 2012.

[4] G. H. Baltuch and M. B. Stern, Deep brain stimulation for Parkinson's disease. CRC Press, 2007.

[5] E. De Momi, C. Caborni, F. Cardinale, G. Casaceli, L. Castana, M. Cossu, R. Mai, F. Gozzo, S. Francione, L. Tassi, G. Lo Russo, L. Antiga, and G. Ferrigno, "Multi-trajectories automatic planner for stereoelectroencephalography (seeg)," International Journal of Computer Assisted Radiology and Surgery, vol. 9, no. 6, pp. 10871097, Nov 2014. [Online]. Available: https://doi.org/10.1007/s11548014-1004-1

[6] J. A. Engh, D. S. Minhas, D. Kondziolka, and C. N. Riviere, "Percutaneous intracerebral navigation by duty-cycled spinning of flexible bevel-tipped needles," Neurosurgery, vol. 67, no. 4, pp. 1117-1123, 2010.

[7] K. B. Reed, A. Majewicz, V. Kallem, R. Alterovitz, K. Goldberg, N. J. Cowan, and A. M. Okamura, "Robot-assisted needle steering," IEEE robotics \& automation magazine, vol. 18, no. 4, pp. 35-46, 2011.

[8] R. Secoli and F. R. y Baena, "Adaptive path-following control for bio-inspired steerable needles," in Biomedical Robotics and Biomechatronics (BioRob), 2016 6th IEEE International Conference on. IEEE, 2016, pp. 87-93.

[9] A. Leibinger, M. J. Oldfield, and F. R. y Baena, "Minimally disruptive needle insertion: a biologically inspired solution," Interface focus, vol. 6 , no. 3, p. 20150107, 2016.
[10] A. Favaro, L. Cerri, S. Galvan, F. Rodriguez y Baena, and E. De Momi, "Automatic optimized 3d path planner for steerable catheters with heuristic search and uncertainty tolerance," in Robotics and Automation (ICRA), 2018 IEEE International Conference on. IEEE, 2018.

[11] J. D. Gammell, S. S. Srinivasa, and T. D. Barfoot, "Batch informed trees (bit*): Sampling-based optimal planning via the heuristically guided search of implicit random geometric graphs," in Robotics and Automation (ICRA), 2015 IEEE International Conference on. IEEE, 2015, pp. 3067-3074.

[12] S. Bériault, F. Al Subaie, D. L. Collins, A. F. Sadikot, and G. B. Pike, "A multi-modal approach to computer-assisted deep brain stimulation trajectory planning," International journal of computer assisted radiology and surgery, vol. 7, no. 5, pp. 687-704, 2012.

[13] D. Scorza, E. De Momi, L. Plaino, G. Amoroso, G. Arnulfo, M. Narizzano, L. Kabongo, and F. Cardinale, "Retrospective evaluation and seeg trajectory analysis for interactive multi-trajectory planner assistant," International Journal of Computer Assisted Radiology and Surgery, vol. 12, no. 10, pp. 1727-1738, 2017.

[14] A. Favaro, A. Lad, D. Formenti, D. D. Zani, and E. De Momi, "Straight trajectory planning for keyhole neurosurgery in sheep with automatic brain structure segmentation," in SPIE Medical Imaging. International Society for Optics and Photonics, 2017, pp. $101352 \mathrm{E}-101$ 352E.

[15] V. Duindam, J. Xu, R. Alterovitz, S. Sastry, and K. Goldberg, "Threedimensional motion planning algorithms for steerable needles using inverse kinematics," The International Journal of Robotics Research, vol. 29, no. 7, pp. 789-800, 2010.

[16] W. Park, J. S. Kim, Y. Zhou, N. J. Cowan, A. M. Okamura, and G. S. Chirikjian, "Diffusion-based motion planning for a nonholonomic flexible needle model," in Robotics and Automation, 2005. ICRA 2005. Proceedings of the 2005 IEEE International Conference on. IEEE, 2005, pp. 4600-4605.

[17] F. Liu, A. Garriga-Casanovas, R. Secoli, and F. R. y Baena, "Fast and adaptive fractal tree-based path planning for programmable bevel tip steerable needles," IEEE Robotics and Automation Letters, vol. 1, no. 2, pp. 601-608, 2016.

[18] S. Patil and R. Alterovitz, "Interactive motion planning for steerable needles in 3d environments with obstacles," in Biomedical Robotics and Biomechatronics (BioRob), 2010 3rd IEEE RAS and EMBS International Conference on. IEEE, 2010, pp. 893-899.

[19] C. Caborni, S. Y. Ko, E. De Momi, G. Ferrigno, and F. R. y Baena, "Risk-based path planning for a steerable flexible probe for neurosurgical intervention," in Biomedical Robotics and Biomechatronics (BioRob), 2012 4th IEEE RAS \& EMBS International Conference on. IEEE, 2012, pp. 866-871.

[20] S. Klein, M. Staring, K. Murphy, M. A. Viergever, and J. P. Pluim, "Elastix: a toolbox for intensity-based medical image registration," IEEE transactions on medical imaging, vol. 29, no. 1, pp. 196-205, 2010.

[21] "Nac brain atlas 2015." [Online]. Available: http://slicer.kitware.com/midas3/item/121588

[22] B. Fischl, "Freesurfer," Neuroimage, vol. 62, no. 2, pp. 774-781, 2012.

[23] R. Viviani, "A digital atlas of middle to large brain vessels and their relation to cortical and subcortical structures," Frontiers in neuroanatomy, vol. 10, 2016.

[24] J. S. William, A. Z. Jonathan, and E. L. William, "Decimation of triangle mesh," Computer Graphics (Proceedings of SIGGRAPH92), pp. 217-226, 1992.

[25] K. Toyoda, E. Urasaki, T. Umeno, W. Sakai, A. Nagaishi, S. Nakane, T. Fukudome, and Y. Yamakawa, "The effectiveness of the stereotactic burr hole technique for deep brain stimulation," Neurologia medicochirurgica, vol. 55, no. 9, pp. 766-772, 2015.

[26] J. A. Bevan, J. Dodge, C. L. Walters, T. Wellman, and R. D. Bevan, "As human pial arteries (internal diameter 200-1000 $\mu \mathrm{m}$ ) get smaller, their wall thickness and capacity to develop tension relative to their diameter increase," Life sciences, vol. 65, no. 11, pp. 1153-1161, 1999.

[27] W. Sun, S. Patil, and R. Alterovitz, "High-frequency replanning under uncertainty using parallel sampling-based motion planning," IEEE Transactions on Robotics, vol. 31, no. 1, pp. 104-116, 2015.

[28] O. Khatib, "Real-time obstacle avoidance for manipulators and mobile robots," The international journal of robotics research, vol. 5 , no. 1 , pp. 90-98, 1986. 